\title{
Power Delay Profile and Noise Variance Estimation for OFDM
}

\author{
Tao Cui, Student Member, IEEE and Chintha Tellambura, Senior Member, IEEE
}

\begin{abstract}
In this letter, we present cyclic-prefix (CP) based noise-variance and power-delay-profile estimators for Orthogonal Frequency Division Multiplexing (OFDM) systems. Signal correlation due to the use of the $\mathrm{CP}$ is exploited without requiring additional pilot symbols. A heuristic estimator and a class of approximate maximum likelihood (ML) estimators are proposed. The proposed algorithms can be applied to both unitary and non-unitary constellations. These algorithms can be readily used for applications such as minimum mean-square error (MMSE) channel estimation.
\end{abstract}

Index Terms-OFDM, channel estimation, SNR estimation.

\section{INTRODUCTION}

$\mathbf{N}$ OISE variance or (equivalently) signal to noise ratio (SNR) is an important measure of channel quality. Their estimation is hence required in many communication applications such as adaptive modulation, turbo coding and others. Several SNR estimation algorithms have been proposed for systems using unitary constellations (i.e., binary phase shift keying (BPSK) and quaternary phase shift keying (QPSK)) over AWGN channels [1], [2]. They can be classified as dataaided (DA), which requires pilot symbols, and non-data aided (NDA) estimators, which do not. In [3], an NDA estimator is extended to systems with non-unitary constellations over Rayleigh fading channels.

In orthogonal frequency division multiplexing (OFDM) systems, noise variance and power delay profile (PDP) are needed for many algorithms such as minimum mean-square error (MMSE) channel estimation and ML frequency offset estimation. In [4], a noise-variance estimator is proposed that directly uses the receiver statistics. A subspace approach is presented in [5] that uses the sample covariance matrix of the received signal. However, both algorithms are DA estimators, which constitute a bandwidth loss. The estimation of the number of multipath gains and associated time delays has been proposed in [6], where pilot symbols are also needed, and channel multipath power and noise variance are required. In [7], a noise variance and SNR estimator that uses training symbols is developed for multiple antenna OFDM systems. Except for these contributions, to the best of our knowledge,

Manuscript received June 8, 2005. The associate editor coordinating the review of this letter and approving it for publication was Prof. George K. Karagiannidis. This work has been supported in part by the Natural Sciences and Engineering Research Council of Canada, Informatics Circle of Research Excellence, and the Alberta Ingenuity Fund.

Tao Cui was with the Dept. of Electrical and Computer Engineering, University of Alberta, Edmonton, Canada. He is now with the California Institute of Technology, CA, USA.

C. Tellambura is with the Dept. of Electrical and Computer Engineering, University of Alberta, Edmonton, Canada (e-mail: chintha@ece.ualberta.ca). Digital Object Identifier 10.1109/LCOMM.2006.01006. no other NDA noise variance and PDP estimators for OFDM systems have been published to date.

In this letter, we develop noise-variance and PDP estimators for OFDM systems over multipath fading channels; the key is to use the fact that the cyclic prefix contains the repeated samples which will introduce a special correlation structure on the received samples. The noise variance, the number of multipath taps, multipath time delays and powers are jointly estimated without pilots. The maximum likelihood (ML) function for the estimated parameters is derived, resulting in an ML estimator.

\section{NOISE VARIANCE AND PDP EstimatoR}

In OFDM, source data are grouped and mapped into $X_{k} \in \mathcal{Q}$, where $\mathcal{Q}$ is a complex signal constellation, and $E\left\{\left|X_{k}\right|^{2}\right\}=1$. Complex data are modulated by inverse discrete Fourier transform (IDFT) on $N$ parallel subcarriers. The symbol interval and block interval are denoted by $T_{s}$ and $N T_{s}$. The resulting OFDM symbol during the $m$ th block interval that comprises $N$ samples is given by

$$
x_{n}(m)=\frac{1}{N} \sum_{k=0}^{N-1} X_{k}(m) e^{\jmath(2 \pi k n / N)}, n=0,1,2, \cdots, N-1 .
$$

The guard interval, inserted to prevent inter-block interference, includes a cyclic prefix that replicates the end of the IFFT output samples. The number of samples in the guard interval $N_{g}$ is assumed to be larger than the delay spread of the channel. The signal is transmitted over a multipath fading channel given by

$$
h(t)=\sum_{l=0}^{L-1} h_{l} \delta\left(t-\tau_{l}\right)
$$

where $L$ is the total number of multipaths, $h_{l} \sim \mathcal{C N}\left(0, \sigma_{l}^{2}\right)$, and $\tau_{l}$ is the delay of the $l$-th path. The received signal after sampling is given by

$$
y_{n}(m)=\sum_{l=0}^{L-1} h_{l} x_{n-d_{l}}(m)+w_{n}(m)
$$

where $w_{n} \sim \mathcal{C N}\left(0, \sigma^{2}\right)$ is an Additive White Gaussian Noise (AWGN), and $d_{l}=\left\lfloor\tau_{l} / T_{s}\right\rceil$ is the delay normalized by $T_{s}$. For simplicity, we round $d_{l}$ to an integer without considering leakage. However, the correlation approach in this paper may also be extended to fractional $d_{l}$. We assume perfect synchronization, and that the channel is invariant within each OFDM block. If there exists a synchronization error, a decision directed algorithm may be applied using our 
proposed parameter estimators and the joint ML time and frequency offset estimator in [8].

At the border between two OFDM blocks $\left(-N_{g} \leq n<0\right)$, the received signal samples can be written as

$$
\begin{aligned}
y_{n}(m) & =\sum_{l=0}^{L-1} h_{l} x_{n-d_{l}}(m) U\left(n-d_{l}\right) \\
& +\sum_{l=0}^{L-1} h_{l} x_{N+n-d_{l}}(m-1) U\left(d_{l}-n\right)+w_{n}(m)
\end{aligned}
$$

where $U(\cdot)$ is the step function. The correlation between each received signal sample over the $\mathrm{CP}$ interval and its corresponding sample at the end of the OFDM block can thus be given by

$$
\begin{aligned}
& E\left\{y_{-k}(m) y_{N-k}^{*}(m)\right\} \\
& =\left\{\begin{array}{cc}
\sigma_{y}^{2}+\sigma^{2} & 0<k \leq N_{g}-d_{L-1} \\
\sum_{l=0}^{L-1} \sigma_{l}^{2} U\left(N_{g}-k-d_{l}\right) & N_{g}-d_{L-1}<k \leq N_{g}-d_{0} \\
0 & N_{g}-d_{0}<k \leq N_{g}
\end{array}\right.
\end{aligned}
$$

where $\sigma_{y}^{2}=\sum_{l=0}^{L-1} \sigma_{l}^{2}$, and $k=1, \ldots, N_{g}$. The expectation in (5) is taken with respect to both $h_{l}$ and $x_{n}(m)$.

When $L$ is large, $y_{n}(m)$ can be modelled approximately as complex Gaussian using the central limited theorem, and the probability density function (pdf) is given by

$$
f\left(y_{n}(m)\right)=\frac{\exp \left(-\frac{\left|y_{n}(m)\right|^{2}}{\sigma_{y}^{2}+\sigma^{2}}\right)}{\pi\left(\sigma_{y}^{2}+\sigma^{2}\right)} .
$$

Samples $y_{-k}(m)$ and $y_{N-k}(m)$ are jointly Gaussian with pdf

$$
\begin{aligned}
& f\left(y_{-k}(m), y_{N-k}(m)\right) \\
= & \frac{\exp \left(-\frac{\left|y_{-k}(m)\right|^{2}+\left|y_{N-k}(m)\right|^{2}-2 \rho_{k} \Re\left\{y_{-k}(m) y_{N-k}^{*}(m)\right\}}{\left(\sigma_{y}^{2}+\sigma^{2}\right)\left(1-\rho_{k}^{2}\right)}\right)}{\pi^{2}\left(\sigma_{y}^{2}+\sigma^{2}\right)\left(1-\rho_{k}^{2}\right)}
\end{aligned}
$$

where

$$
\begin{aligned}
\rho_{k} & =\left|\frac{E\left\{y_{-k}(m) y_{N-k}^{*}(m)\right\}}{\sqrt{E\left\{|y-k(m)|^{2}\right\} E\left\{\left|y_{N-k}(m)\right|^{2}\right\}}}\right| \\
& =\frac{\sum_{l=0}^{L-1} \sigma_{l}^{2} U\left(N_{g}-k-d_{l}\right)}{\sum_{l=0}^{L-1} \sigma_{l}^{2}+\sigma^{2}} .
\end{aligned}
$$

Therefore, the proposed estimator is only approximate ML.

We use $M$ OFDM blocks to estimate those parameters and assume that they remain unchanged during the $M$ blocks. Define $\mathbf{p}=\left[\sigma_{0}^{2}, \ldots, \sigma_{L-1}^{2}\right], \mathbf{d}=\left[d_{0}, \ldots, d_{L-1}\right]$ and $\mathbf{y}=$ $\left[y_{-N_{g}}(1), y_{-N_{g}+1}(1), \ldots, y_{N-1}(M)\right]$. Using (6) and (7) and assuming the $M$ OFDM blocks are independent, the loglikelihood function of $\mathbf{y}$ conditioned on $\sigma^{2}, \mathbf{p}, \mathbf{d}$ can be written as

$$
\begin{aligned}
& \Lambda\left(\mathbf{y} \mid \sigma^{2}, \mathbf{p}, \mathbf{d}\right) \\
= & \sum_{m=1}^{M} \log \left(\prod_{k=1}^{N_{g}} f\left(y_{-k}(m), y_{N-k}(m)\right) \prod_{k=0}^{N^{\prime}} f\left(y_{k}(m)\right)\right) \\
= & -M\left(\sum_{k=1}^{N_{g}} \frac{a_{k}-\rho_{k} b_{k}}{c\left(1-\rho_{k}^{2}\right)}+\log \left(c\left(1-\rho_{k}^{2}\right)\right)+\sum_{k=0}^{N^{\prime}} \frac{g_{k}}{c}+\log (c)\right)
\end{aligned}
$$

where $N^{\prime}=N-N_{g}-1$,

$$
\begin{aligned}
a_{k} & =\frac{\sum_{m=1}^{M}\left|y_{-k}(m)\right|^{2}+\left|y_{N-k}(m)\right|^{2}}{M} \\
b_{k} & =\frac{\sum_{m=1}^{M} \Re\left\{y_{-k}(m) y_{N-k}^{*}(m)\right\}}{M} \\
g_{k} & =\frac{\sum_{m=1}^{M}\left|y_{k}(m)\right|^{2}}{M}, c=\sigma_{y}^{2}+\sigma^{2} .
\end{aligned}
$$

Since (9) involves many variables, to simplify the joint parameters' estimation, we take a suboptimal way. We first estimate $c$ by maximizing only the last sum in (9):

$$
\hat{c}=\frac{\sum_{k=0}^{N^{\prime}} g_{k}}{N-N_{g}}=\frac{\sum_{k=0}^{N^{\prime}} \sum_{m=1}^{M}\left|y_{k}(m)\right|^{2}}{\left(N-N_{g}\right) M} .
$$

From (11), we find $\hat{c}$ is the time average estimation of $\sigma_{y}^{2}+\sigma^{2}$, and hence an estimate of $c$ is given by $\hat{c}$. Substituting $\hat{c}$ back into the first summation of (9) and maximizing $\rho_{k}$ individually, we get the estimate for $\rho_{k}$ as the real root of the equation

$$
2 \hat{c} \rho^{3}-b \rho^{2}-2\left(\hat{c}-a_{k}\right) \rho-b=0 .
$$

We then compute the value $s_{k}$ as

$$
s_{k}=\left\{\begin{array}{cc}
\rho_{N_{g}} \hat{c} & k=1 \\
\left(\rho_{N_{g}-k+1}-\rho_{N_{g}-k+2}\right) \hat{c} & k=2, \ldots, N_{g}
\end{array} .\right.
$$

A threshold value is set as $\alpha \hat{c}$, where $\alpha$ is a constant less than 1. If $s_{k}>\alpha \hat{c}$, it is identified as a path; $s_{k}$ is the estimate of path power, and $k$ is the estimate of delay time. The number of paths is estimated as the number of $s_{k}$ that $s_{k}>\alpha \hat{c}$. We denote the maximum delay time as $d_{\max }$. The noise variance can thus be estimated as

$$
\hat{\sigma}^{2}=\hat{c}\left(1-\frac{\sum_{k=1}^{N_{g}-d_{\max }+1} \hat{\rho}_{k}}{N_{g}-d_{\max }+1}\right) .
$$

If we look directly at the structure of OFDM block, in the absence of noise, $y_{-k}(m)=y_{N-k}(m)$ for $k=1, \ldots, N_{g}-$ $d_{\max }+1$. The noise variance can be obtained alternatively as

$$
\hat{\sigma}^{2}=\frac{\sum_{m=1}^{M} \sum_{k=1}^{N_{g}-d_{\max }+1}\left|y_{N-k}(m)-y_{-k}(m)\right|^{2}}{2 M\left(N_{g}-d_{\max }+1\right)} .
$$

Using the results of (11) and (14) or (15), SNR can be estimated by

$$
\mathrm{SNR}=\frac{\hat{c}}{\hat{\sigma}^{2}} .
$$

Note that (15) can only be used to estimate $\sigma^{2}$.

Note that the SNR considered in this paper is the average SNR ( averaged over the channel, data and noise realizations). Our proposed algorithm cannot estimate the instantaneous SNR, where a fixed channel is considered.

\section{Simulation Results}

We now investigate the performance of our proposed estimators. We assume an OFDM system using QPSK with $N=64$ subcarriers, and CP length $N_{g}=16$. A $L=$ 6 channel model is used. The power profile is given by $\mathbf{p}=[0.189,0.379,0.239,0.095,0.061,0.037]$, and the delay profile after sampling is $\mathbf{d}=[0,1,2,4,6,8]$. Each path is an independent, zero-mean complex Gaussian random process. 


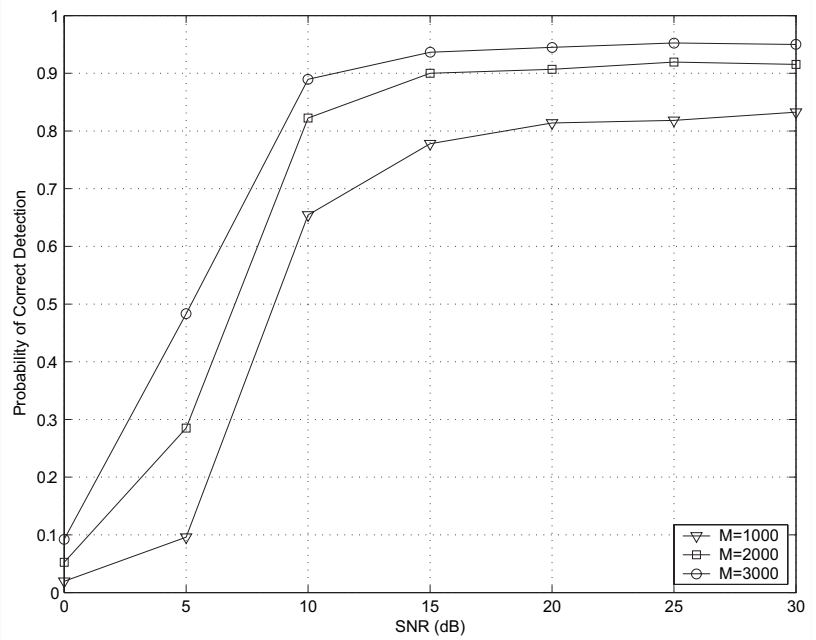

Fig. 1. The probability of correct detection of the number of paths.

Fig. 1 shows the probability of correct detection of the number of paths using our proposed algorithm with different $M$. The threshold parameter is set to $\alpha=0.01$. In low SNR, the paths with smaller power are dominated by the noise, and there may be many paths larger than the threshold. Therefore, the number of paths may be overestimated. The probability of correct detection increases in high SNR. With increasing $M$, the probability of detection error decreases.

Fig. 2 presents the normalized mean square error (NMSE) of the channel power estimation for the 3rd path (arbitrarily chosen), where the NMSE is defined as NMSE $=E\left\{\left(\hat{\sigma}_{3}^{2}-\right.\right.$ $\left.\left.\sigma_{3}^{2}\right)^{2}\right\} / \sigma_{3}^{4}$. The channel power is overwhelmed by the noise in low SNR. In high SNR, the NMSE becomes constant since the number of paths cannot be $100 \%$ correctly detected. The NMSE is improved by increasing $M$.

Fig. 3 shows the NMSE of the noise variance estimation using different estimators, where the NMSE is defined as NMSE $=E\left\{\left(\hat{\sigma}^{2}-\sigma^{2}\right)^{2}\right\} / \sigma^{4}$. The estimator using (14) is denoted as ML, and that using (15) is denoted as direct estimator or (DI). At low SNR, the DI method performs better than the approximate ML method since the probability of $d_{\max }$ detection is higher for the DI method. In high SNR, both DI and ML perform identically. With the increase of $M$, the performance of both estimators improve.

\section{CONCLUSION}

In this paper, we have presented noise-variance and powerdelay-profile estimators using the CP in each OFDM block. The correlation structure due to the use of the CP has been exploited to derive our estimators, and hence pilot symbols are not needed. A direct heuristic noise variance estimator has also been proposed. Simulation results show that our proposed estimators provide an effective way to estimate the channel parameters. The results in this paper may be used to improve the performance and reduce the complexity of channel estimators for OFDM systems.

\section{REFERENCES}

[1] N. C. Beaulieu, A. S. Toms, and D. R. Pauluzzi, "Comparison of four SNR estimators for QPSK modulations," IEEE Commun. Lett., vol. 4, pp. 43-45, Feb. 2000.

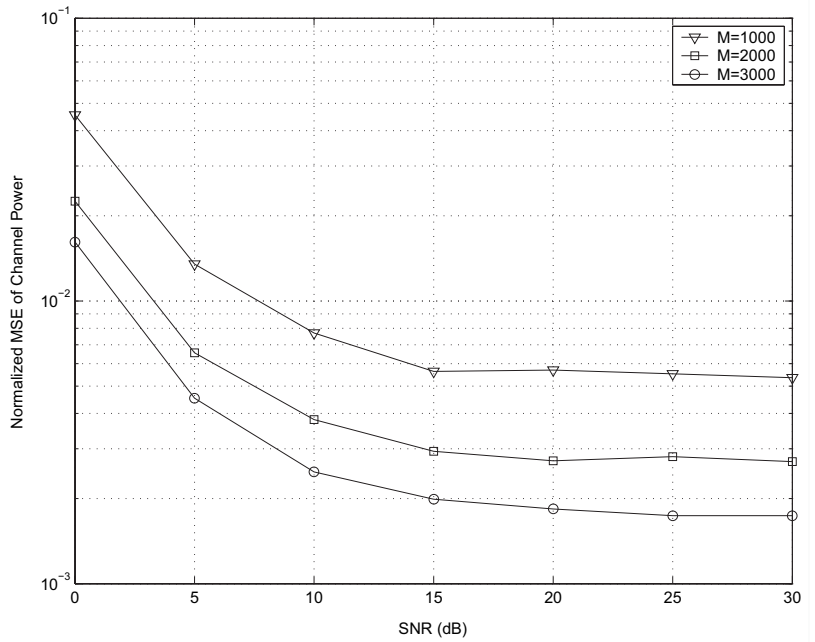

Fig. 2. The NMSE of the channel power estimation for the 3rd path.

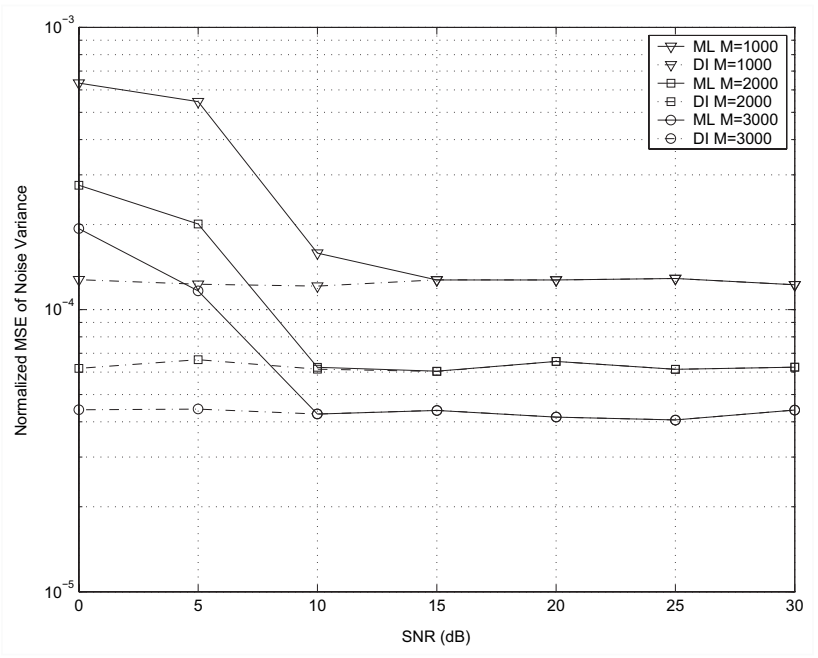

Fig. 3. The NMSE of the noise variance estimation using different estimators.

[2] N. S. Alagha, "Cramer-Rao bounds of SNR estimates for BPSK and QPSK modulated signals," IEEE Commun. Lett., vol. 5, pp. 10-12, Jan. 2001.

[3] P. Gao and C. Tepedelenlioglu, "SNR estimation for nonconstant modulus constellations," IEEE Trans. Signal Processing, vol. 53, pp. 865-870, Mar. 2005.

[4] S. He and M. Torkelson, "Effective SNR estimation in OFDM system simulation," in Proc. GLOBECOM, Nov. 1998, pp. 945-950.

[5] X. Xu, Y. Jing, and X. Yu, "Subspace-based noise variance and SNR estimation for OFDM systems," in Proc. WCNC, Mar. 2005, pp. 23-26.

[6] B. Yang, K. B. Letaief, R. S. Cheng, and Z. Cao, "Channel estimation for OFDM transmission in multipath fading channels based on parametric channel modeling," IEEE Trans. Commun., vol. 49, pp. 467-479, Mar. 2001.

[7] S. Boumard, "Novel noise variance and SNR estimation algorithm for wireless MIMO OFDM systems," in Proc. GLOBECOM, Dec. 2003, pp. 1330-1334.

[8] J. J. van de Beek, M. Sandell, and P. O. Borjesson, "ML estimation of time and frequency offset in OFDM systems," IEEE Trans. Signal Processing, vol. 45, pp. 1800-1805, July 1997. 